\section{Identification of the source of permanent glare from a three-piece IOL}

${ }^{1}$ Moseley, Birmingham, UK

${ }^{2}$ Division of Physics, Center for Devices and Radiological Health, FDA, Rockville, $M D$, USA

${ }^{3}$ St Luke Eye Institute, Amarillo, TX, USA

Correspondence: RJ Landry, Division of Physics, HFZ-13, Center for Devices and Radiological Health, FDA, 9200 Corporate Boulevard, Rockville,

MD 20850, USA

Tel: + 13018274687 ;

Fax: +1 3017274677

E-mail: robert.landry@

fda.hhs.gov

Received: 27 January 2006 Accepted: 24 April 2006 Published online: 25 August 2006

Financial/proprietary interest: The authors do not have any commercial or proprietary interest in any of the products referred to in this paper

\begin{abstract}
Objective To identify the source of unwanted glare images from a three-piece intraocular lens (IOL) implant following cataract surgery. Method The IOL and posterior capsule were examined under mydriatic and nonmydriatic conditions using direct focal illumination from a slit lamp biomicroscope. Direct focal illumination was undertaken with both a narrow beam $(0.1 \mathrm{~mm}$ in width) and small spot $(0.1 \mathrm{~mm}$ in diameter) to identify the points at which the glare images were stimulated. While observing the location of the beam with the slit lamp biomicroscope, the patient indicated when the glare images were stimulated.

Results The nasal haptic insertion into the optic was identified as the source of temporal line images arising from lights such as headlamps from oncoming cars and street lamps. The adjacent edge of the IOL was also identified as the likely source of additional cob web-like light rays.

Conclusions The haptic insertions in threepiece IOLs may, under certain conditions, interfere with light entering the pupil and produce extraneous images. Large mesopic pupils and decentred IOLs are conditions that increase the likelihood of unwanted glare images.

Eye (2007) 21, 1078-1082; doi:10.1038/sj.eye.6702539; published online 25 August 2006
\end{abstract}

Keywords: haptic-glare; line glare image; diffuse glare image; three-piece intraocular lenses

\section{Introduction}

The phenomenon of glare with intraocular lens implants (IOLs) has been reported for some time. ${ }^{1-17}$ Mamalis, in 1998, surveyed the complications associated with foldable IOLs that required explantation or secondary intervention. ${ }^{7}$ Glare and optical aberrations were identified as the cause in $13 \%$ of the cases where three piece, silicone, monofocal lenses had been used and in $44 \%$ of the cases involving three piece acrylic lenses. ${ }^{7}$ Mamalis also noted that these symptoms were sometimes severe enough to require removal or exchange of these IOLs.

Disturbing images from IOLs have been reported in a number of papers in recent years. ${ }^{6-17}$ In one recent study involving 6668 patients fitted with PMMA monofocal IOLs, $20 \%$ experienced some degree of transient glare. ${ }^{9}$ Thirteen patients experienced permanent glare. The glare was so severe in five cases that IOL explantation and replacement was required.

In another study involving 415 patients, the incidence of permanent glare with two different models of monofocal acrylic IOLs ranged from 0.5 to $2.2 \% .{ }^{14}$ One-third of those experiencing glare required explantation and replacement of their IOLs.

The findings from these two studies suggest that roughly between 0.2 and $2 \%$ of patients undergoing cataract surgery with monofocal IOL implantation experience permanent glare. Assuming that there are about 1.5 million cataract surgeries per year in the US, these studies suggest that between 3000 and 30000 patients per year in the US experience permanent glare following IOL implantation. The two studies cited above suggest that roughly somewhere between 1000 and 10000 patients have their IOLs explanted and replaced each year. The remaining patients may tolerate living with the symptoms. Therefore, although the incidence of permanent glare following cataract surgery and IOL implantation is small, the total number of patients affected is significant.

In this paper we describe a method of identifying the precise source of permanent glare in a patient with a three piece silicone IOL along with the results obtained. 


\section{Background}

A 67-year-old male had a cataractous lens removed from his left eye followed by implantation of a three piece, 18D, $6 \mathrm{~mm}$ diameter, foldable, silicone IOL with bluecoloured haptics. The edge of this IOL is flat with rounded corners. The surgery was performed through a $3.5 \mathrm{~mm}$ clear corneal incision. Postoperatively, the patient was treated with topical antibiotics and steroid eyedrops. On the day following surgery, the corrected visual acuity was $20 / 25$. Three months later, corrected visual acuity remained unchanged at 20/25.

Three days after surgery, the patient noticed that while driving a car at night each headlamp from oncoming cars was producing a bright line image on either side of an image of the headlamp itself in the upper half of his visual field (Figure 1(a)). A similar bright line image was produced by headlamps reflected from the left side view mirror, but in this case the headlamp image was positioned near the left end of the line image (Figure 1b). In both cases, the line image was tilted approximately 20-30 from the horizontal. When there was a line of cars, there was a series of line images (Figure 1(c)). As the oncoming cars passed by, the line images synchronously moved up and out of the field of view. The patient subjectively estimated the intensity of the line image to be of the order of $10 \%$ of the intensity of the light source itself. Overhead street lamps located temporally to the left eye produced a similar line image to that produced by oncoming headlamps.

Approximately 7 weeks after surgery, the patient noticed that he could see thin sliver-like light rays in the right lower quadrant of the visual field of his left eye
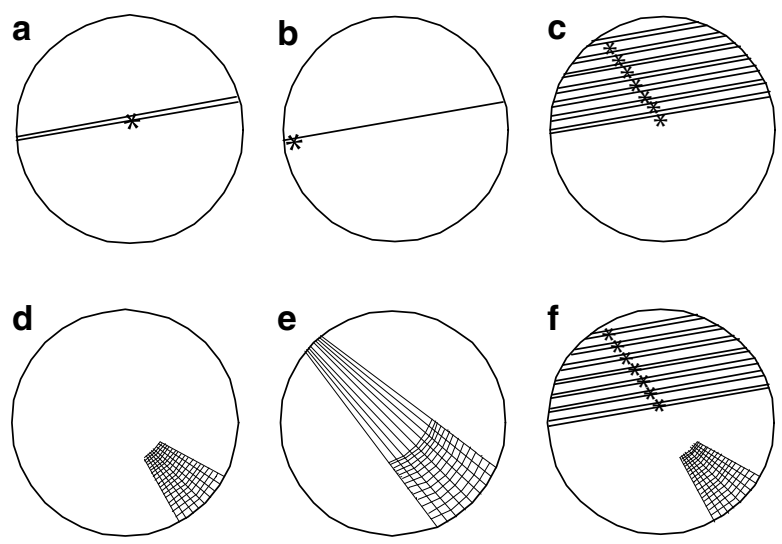

Figure 1 Schematic drawings of glare images formed from: (a) oncoming headlamps of a car, (b) reflection of the headlamp of a following car from the left rear view mirror, (c) a line of oncoming cars, (d) and (e), an overhead street lamp at a steep angle, and, (f) a line of oncoming cars and overhead street lamp at the angles with respect to the eye that stimulate the glare images, the patient experiences all images at the same time. when passing overhead street lamps positioned at a steep angle $\left(60^{\circ}\right)$ relative to the left eye (Figure $1(\mathrm{~d})$ ). As the angle increased, these rays increased in length eventually covering the field from top to bottom (Figure 1(e)). Finally, when a line of cars and overhead lamps were seen at the same time in the positions described, the patient experienced all images simultaneously (Figure 1(f)).

Three months following surgery, the surgeon noted patchy fibrosis on the posterior capsule but it did not contain any folds, tension lines, or striae. He concluded, therefore, that the posterior capsule did not appear to be the source of the glare images. Three years after surgery, the patient reported no significant change in the glare images.

\section{Methods and results}

The patient was examined independently by two other clinicians to try to identify the source of the glare images - the first at 4 months, the other at 11 months following surgery. It was noted that the pupils were unusually large for a 67-year-old subject. At the 11-month postoperative visit, the pupils were measured with the Rosenbaum Pocket Vision Screener. They were found to be $4.5 \mathrm{~mm}$ in the right eye and $5.0 \mathrm{~mm}$ in the left under mesopic conditions and $4 \mathrm{~mm}$ in both eyes under photopic conditions. The pupil reactions, both direct and consensual, were normal both to light and convergence.

With the slit lamp beam positioned close to the visual axis, the insertion of the nasal haptic into the implant was visible tangential to the pupil margin. The temporal insertion, on the other hand, could only be seen with the microscope positioned at a very oblique angle on the nasal side of the visual axis. This would indicate that the implant, relative to the pupil, was not precisely centred. It was also noted that the haptic insertions were not vertical but were at an angle of about $75^{\circ}$, that is $15^{\circ}$ away from the vertical, along the $7 \mathrm{O}^{\prime}$ clock-1 O'clock axis (Figure 2). The edge of the IOL was covered by the remaining, but completely opaque, anterior capsule.

During examination of the implant using direct focal illumination with a narrow beam $(0.1 \mathrm{~mm}$ in width) at both 4 and 11 months postoperatively, the patient observed that he was occasionally aware of a line image in the upper half of his visual field. When the slit beam was increased in width this image disappeared. In an attempt to locate the source of the line image, the pupillary area was scanned using the $0.1 \mathrm{~mm}$ beam. As the slit beam clipped the edge of the haptic insertion, the patient observed that the line image was faintly visible. It became clearly visible as the insertion became fully illuminated. In order to verify that the haptic insertion was the source of the line image, the beam was truncated 



Figure 2 Schematic drawing of IOL (large circle with J loops) and mesopic $5 \mathrm{~mm}$ diameter pupil (small circle) under the conditions: (a) IOL centred on the pupil, (b) IOL decentred by $0.5 \mathrm{~mm}$ temporally to the pupil, and, (c) the IOL decentred by $0.5 \mathrm{~mm}$ temporally to the pupil and rotated to a $7 \mathrm{O}^{\prime}$ clock-1 $\mathrm{O}^{\prime}$ clock position as in the eye of the patient.

to produce a circular spot with a diameter of $0.1 \mathrm{~mm}$, thus ensuring that the spot could be focused and contained exactly within the insertion (see the $\mathrm{X}$ mark in Figure 2(c)). When the beam was truncated, the patient described the appearance of the image as being identical to that which he had previously seen from car headlamps. Projecting the spot across the posterior capsule, however, did not produce any sensation of light or glare, notwithstanding the fact that fibrosis was present.

In order to be certain that the line image was being produced by the haptic insertion, the clinician's slit lamp observations were used to identify the points at which the patient could see the line images. As the haptic insertion became minimally illuminated, the clinician observed that the line image should be just visible with its intensity increasing as the haptic became fully illuminated. The patient subsequently confirmed these observations as correct. The procedure was repeated, with the patient again confirming the accuracy of the clinician's description. These results clearly indicate that the haptic insertion is the source of the line image.

While it was not possible to arrange the slit lamp beam to simulate an overhead street lamp, it was possible to simulate such conditions using a hand held spotlight. This was achieved by reducing the room illumination to a mesopic level and then switching on the spotlight. The spotlight was positioned just forward of the patient's eye and then rotated from the $6 \mathrm{O}^{\prime}$ clock to the $12 \mathrm{O}^{\prime}$ clock position. When the light was positioned below the patient's eye, no extraneous images were seen. However, as the spotlight approached the $12 \mathrm{O}^{\prime}$ clock position above the eye, sliver-like rays became faintly visible in the inferior nasal quadrant of the visual field. The patient

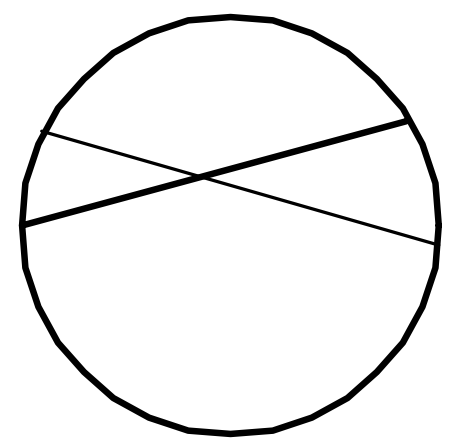

Figure 3 Schematic drawing of glare image formed when a point source is directed at the junction of the nasal haptic and fibrosed tissue on the anterior capsule.

described them as becoming increasingly prominent as the spotlight was moved towards the $1 \mathrm{O}^{\prime}$ clock position and beyond, that is, into the superior temporal quadrant in front of the left eye-the identical position of the offending street lights.

The eye was re-examined with a slit lamp following the administration of a mydriatic. A $360^{\circ}$, circular, continuous capsulorrhexis was about $5.5 \mathrm{~mm}$ in diameter. The IOL with blue coloured haptics was completely in the capsule. Examination also confirmed that the IOL was decentred with the haptics tilted along a 7 O'clock-1 $\mathrm{O}^{\prime}$ clock axis. Using the smallest slit lamp beam as a measuring device, the horizontal decentration appeared to be approximately $0.5 \mathrm{~mm}$.

When the $0.1 \mathrm{~mm}$ diameter slit lamp beam was focused through the dilated pupil onto the visible tips of the haptic in the optic, the patient once again described seeing a line image. The line originating from the haptic on the nasal side was more distinct than that originating from the temporal side. While scanning the nasal haptic rod with the $0.1 \mathrm{~mm}$ diameter beam, the patient continued to see the line until the edge of the opacified anterior capsule was reached, at which point the patient described the light as appearing as an ' $x$ ' rather than a line image (Figure 3).

\section{Discussion and conclusions}

The 67-year-old patient involved in this case has unusually large $5 \mathrm{~mm}$ diameter pupils under mesopic conditions. The implant used was a $6 \mathrm{~mm}$ diameter, three piece, foldable, silicone IOL. The top ends of the haptic insertions are each inset $0.5 \mathrm{~mm}$ from the edge of the implant and are, therefore, spaced $5 \mathrm{~mm}$ apart. If such an IOL is perfectly and exactly centred in an eye with a $5 \mathrm{~mm}$ diameter mesopic pupil, the top of the haptics in the optic would be located right at the edge of the patient's pupil as shown in Figure 2(a). In this situation, 
it is clear that the haptic insertions may, under certain conditions, interfere with light entering the pupil and produce extraneous images.

In this case, however, the IOL is not exactly centred. The IOL is decentred relative to the pupil, about $0.5 \mathrm{~mm}$ temporally. As a result, the top of the nasal haptic insertion is sited within the pupillary area. (Figure 2(b) and (c)). The patient is experiencing disturbing extraneous line images in his field of vision. This investigation has identified the nasal haptic insertion into the optic as being the source of these images.

On the basis of the findings in this study, it is also possible to determine the likely cause of the additional sliver-like rays associated with street lamps that the patient occasionally experiences. These rays appear to the patient to be positioned in the lower nasal quadrant of the field of the left eye. This appearance occurs when the street lamp is positioned, in the superior temporal field of the left eye. Under normal circumstances, one would expect all the rays from an overhead street lamp to be identified as originating in the superior field. The fact that the sliver-type rays appear to the patient to be originating in the lower nasal quadrant of the left field, means that some of the light from the street lamp is being reflected back towards the superior temporal retina.

The fact that this phenomenon only became apparent after insertion of the implant clearly indicates that the reflections emanate from a portion of the implant. Under simulated conditions, it was observed that the sliver-like rays of light became increasingly prominent as the spotlight moved past $12 \mathrm{O}^{\prime}$ clock towards the $1 \mathrm{O}^{\prime}$ clock position. With the spotlight in this position, the edge of the IOL near the nasal haptic is illuminated. This suggests that the light is being reflected from the edge of the IOL close to the location where the haptic is inserted into the optic.

A number of points emerge from this work. The patient was fitted with a three piece, $6 \mathrm{~mm}$ optic diameter implant when his mesopic pupil is $5 \mathrm{~mm}$ in diameter. Even if the implant had been centred precisely, the end of the haptic insertion would, at times, encroach into his pupillary area. A small error in centration of as little as $0.5 \mathrm{~mm}$ effectively places not only the insertion, but also the edge of the optic in the pupillary area. The outcome for this patient is the presence of disturbing and unwanted extraneous images reducing the quality of vision.

Serdarevic has addressed the issue of 'How to choose the right IOL for your cataract patient'. ${ }^{17}$ She pointed out that before selecting an appropriate lens, it is important to consider the specific properties and characteristics of different IOLs including the possible sources of glare. In drawing attention to the problems of glare caused by edge and surface reflections, she observed that elderly patients who have small pupils might not experience such glare. This latter point recognizes that it is the pupil size that is a critical factor in determining whether the periphery of the IOL is illuminated, thus producing unwanted reflections and disturbing glare. It is important to bear in mind, however, that not all elderly patients have small pupils. A small percentage of cataract patients, such as the patient described here, will have large pupils.

These results show that measurement of mesopic pupil diameter before surgery allows for an assessment of the likelihood that extraneous images may be experienced from the commonly used $5 \mathrm{~mm}$ or $6 \mathrm{~mm}$ optic diameter, three piece IOLs. For patients with mesopic pupil diameters in excess of $4 \mathrm{~mm}$, consideration might be given to using a three piece IOL with an optic diameter of $6.5 \mathrm{~mm}$ or greater or a single piece IOL, where the haptic does not encroach into the optic. Both of these steps would reduce the likelihood that unwanted images would be experienced.

\section{Disclaimer statement}

The mention of commercial products, their sources, or their use in connection with material reported herein is not to be construed as either an actual or implied endorsement of such products by the United States Department of Health and Human Services.

\section{References}

1 Alpar JJ. Glare factors in different intraocular lenses: Comparative study in patients with dissimilar binocular implants, in Current Concepts in Cataract Surgery, Selected Proceedings of the Seventh Biennial Cataract Surgical Congress, Appleton-Century-Crofts/New York. 1982; 285-290.

2 Rosner M, Shari M, Blumenthal M. Optical aberrations from a well-centered intraocular lens implant. Am J Ophthalmol 1986; 101: 117-118.

3 Landry R. Unwanted optical effects caused by intraocular lens positioning holes. J Cataract Refract Surg 1987; 13: 421-423.

4 Burk PO. Investigation of unwanted images caused by intraocular lenses. J Cataract Refract Surg 1988; 21: 334-338.

5 Masket S, Geraghty E, Crandall AS, Davison JA, Johnson $\mathrm{SH}$, Koch DD et al. Undesired light images associated with ovoid intraocular lenses. J Cataract Refract Surg 1993; 19: 690-694.

6 Holladay JT, Lang A, Portney V. Analysis of edge glare phenomenon in intraocular lens edge designs. J Cataract Refract Surg 1999; 25: 748-752.

7 Mamalis N. Complications of foldable intraocular lenses requiring explantation or secondary intervention-1998 survey. J Cataract Refract Surg 2000; 26: 766-772.

8 Kruger AJ, Schauersberger J, Abela C, Schild G, Amon M. Two year results: sharp versus rounded optic edges on silicone lenses. J Cataract Refract Surg 2000; 26: 566-570. 
9 Davison JA. Positive and negative dysphotopia in patients with acrylic intraocular lenses. J Cataract Refract Surg 2000; 26: 11346-11355.

10 Masket S. Truncated edge design, dysphotopia, and inhibition of posterior capsule opacification. J Cataract Refract Surg 2000; 26: 145-147.

11 Farbowitz MA, Zabriskie NA, Crandall AS, Olson RJ, Miller M. Visual complaints associated with the AcrySof acrylic intraocular lens. J Cataract Refract Surg 2000; 26: 1339-1345.

12 Javitt J, Brauweiler H-P, Jacobi KW, Klemen U, Kohnen S, Quentin C-D et al. Cataract extraction with multifocal intraocular lens implantation: clinical, functional, and quality-of-life outcomes. J Cataract Refract Surg 2000; 26: 1356-1366.
13 Haring G, Dick HB, Krummenauer F, Weissmantel U, Kroncke W. Subjective photic phenomenon with refractive multifocal and monofocal intraocular lenses. J Cataract Refract Surg 2001; 27: 245-249.

14 Ellis MF. Sharp-edged intraocular lens design as a cause of permanent glare. J Cataract Refract Surg 2001; 27: 1061-1064.

15 Erie JC, Bandhauer MH, McLaren JW. Analysis of postoperative glare and intraocular lens design. J Cataract Refract Surg 2001; 27: 614-621.

16 Mamalis N, Spencer TS. Complications of foldable intraocular lenses requiring explantation or secondary intervention-2000 survey update. J Cataract Refract Surg 2000; 27: 1310-1317.

17 Serdarevic O. How to choose the right IOL for your cataract patient. Ophthalmol Times 2002; 21. 